Original Research Article

\title{
A Study of the Right Colonic Vascular Anatomy: Correlations between Veins and Arteries
}

\author{
Nao Obara ${ }^{1)}$, Shigeki Yamaguchi ${ }^{1)}$ and Yoshitaka Okada ${ }^{2)}$ \\ 1) Department of Gastroenterological Surgery, Saitama Medical University International Medical Center, Hidaka, Japan \\ 2) Department of Diagnostic Radiology, Saitama Medical University International Medical Center, Hidaka, Japan
}

\begin{abstract}
Objectives: Few studies have examined the correlations between the arteries and veins of the right colon. In this study, we aimed to use high-resolution CT scans to understand the vascular anatomy of Henle's gastrocolic trunk and review the terminology describing the arteries and veins of the right colon.

Methods: This retrospective study has examined patients who underwent laparoscopic colectomy for right colon cancer in a single institution in Japan. Scans from consecutive patients who underwent surgery between October 2017 and March 2020 ( $\mathrm{n}=165)$ were examined. Preoperative CT images were used to create multiplanar reformation images and volume rendering images.

Results: Among the 139 patients with Henle's gastrocolic trunk (GCT) present, arteries accompanying the accessory right colic vein (ARCV) were most common on the right branch of the middle colic artery (MCA) (71.2\%), followed by the right colic artery (RCA) (19.4\%); meanwhile, 9.4\% of the patients had no accompanying arteries. Of patients with no accompanying arteries to the ARCV, RCA was present in $15.4 \%$. Among the 26 patients with no GCT, the right colic vein (RCV) existed in 15 patients, with the artery accompanying the RCV most commonly being the right branch of the MCA (66.6\%), followed by the RCA $(33.3 \%)$.

Conclusions: Irrespective of the presence of GCT, approximately $70 \%$ of the arteries accompanying the drainage vein from the right colon were the right branch of the MCA. We suggest that vascular branch formation be considered preoperatively in surgical management for right colon cancer.
\end{abstract}

\section{Keywords \\ right colon, artery, vein}

J Anus Rectum Colon 2021; 5(3): 306-312

\section{Introduction}

The standard operations used for the treatment of colorectal cancer are primary lesion resection surgery and systemic lymphadenectomy. Recently, there has been a focus on complete mesocolic excision and central vascular ligation[1-4], in an effort to improve results following colon cancer treatment. In Japan, importance is mostly placed on central resection as D3 resection[5,6]. Nevertheless, it is important that there is sufficient understanding on vascular anatomy to perform central dissections.

For lymph node resection in cases of right colon cancer, the flow of lymph along the surgical trunk is thought to be important. Various studies report its vascular anatomy; however, there are many different branch morphologies, and with blood vessels being referred to by different names in different reports, there are some aspects which are yet to be understood in a unified manner. 
Existing reports have mostly discussed branch morphologies and frequency of occurrence of the various systems of the superior mesenteric arteries and veins[7-20]. However, there are very few reports examining the correlations between the arteries and veins, with reports being limited to those discussing the relationship between the ileocolic artery (ICA) and vein with the superior mesenteric vein[21-26]. In particular, there is no report which expounds on the correlations between Henle's gastrocolic trunk (GCT) and accompanying arteries. GCT, in which veins from the colon, stomach, omentum, and pancreas merge, is present in the right colon[27] and exhibits a large variety of correlations with arteries; yet, the terminology of the colic branches is yet to be unified. During surgical operations, awareness of the three-dimensional correlations between the arteries and veins is thought to improve both safety and accuracy of the procedure. Accordingly, knowing the frequency of occurrence of the various vascular correlation variations is of utmost importance.

Many vascular structures have been historically described in human anatomy, but it has recently become possible to gain an in-depth understanding of the fine details of vascular anatomy due to the development of modern, high-resolution CT scans. For this study, high-resolution scans were used to investigate the correlations between the arteries and veins distributed in the right colon.

\section{Methods}

Using CT images from a single institute, we performed observational research into the vascular morphology of the right colon. The positions and frequency of the right colon's vascular system, particularly the ARCV, and morphology and frequencies of the branches of GCT (namely, colic branch, gastroepiploic branch, and pancreatic branch) were recorded.

\section{Study design}

This research was implemented under an audit by Saitama Medical University International Medical Center IRB and upon approval from the head of the research facility (approval number 18-191). This study was a single-institutional retrospective study that used existing information from hospital medical records. Data which were linkable and anonymized were utilized. Cases where patients had undergone laparoscopic surgery for right colon cancer at the hospital, between October 12017 and March 31 2020, were examined. Preoperative CT images were used to create multiplanar reformation images and volume rendering images, and branch formation on the right colon and correlations were retrospectively analyzed. All patients gave informed consent for their data to be used in future analysis.

64-slice (LightSpeed VCT, GE Healthcare, Chicago, Illi- nois, USA) or 320-slice (Aquilion ONE, Canon Medical Systems, Tochigi, Japan) multi-slice CTs were used for the scans. Following plain CT imaging, $100 \mathrm{~mL}$ of $370 \mathrm{mg} / \mathrm{mL}$ water-soluble iodine contrast agent (or $2 \mathrm{~mL} / \mathrm{kg}$ where patient weight $<50 \mathrm{~kg}$ ) was injected into the cubital vein for over 25 seconds, and images were taken at the early phase, portal vein phase, and the delayed phase. Images were observed using an image analysis volume analyzer (SYNAPSE VINCENT, Fuji Film, Tokyo, Japan). Coronal and sagittal multiplanar reformation images of thickness $0.5 \mathrm{~mm}$ or $0.625 \mathrm{~mm}$ were created, and the branching and positioning of the blood vessels were observed. Volume rendering imaging was created for some cases, and they were used for reference.

Branch morphology of the GCT, placement of the middle colic artery (MCA), right colic artery (RCA) and ICA, and the correlations between them were determined through consensus between an experienced gastrointestinal surgeon and a highly experienced radiologist specializing in the abdominal region. Cases which had standard-quality CT imaging, where CT imaging had been performed at a different hospital, or where detailed analysis of the vascular branches was not possible due to inappropriate timed contrast imaging, were excluded, providing a total of 165 cases for analyses.

\section{Analysis}

Surgical anatomy of the right colon and correlations between arteries and veins were then analyzed and investigated. In particular, morphologies of the arteries accompanying the accessory right colic vein (ARCV) or the right colic vein (RCV) were considered. Furthermore, the branching frequency and morphology of the GCT colic branch, gastroepiploic branch, and pancreatic branch were also considered.

\section{Definition of blood vessel names}

\section{1. $R C A$}

Arteries directly branching from the superior mesenteric artery (SMA) that flow to the ascending colon are referred to as the RCA, whereas arteries branching from the MCA or ICA that flow into the ascending colon are not included under the term RCA.

\section{2. $R C V$}

The vein which flows from the ascending colon directly to the superior mesenteric vein (SMV) is referred to as the RCV.

\section{3. $A R C V$ and $G C T$}

The colic branch, which forms a common trunk with the right gastroepiploic vein (RGEV), is referred to as the ARCV; their common trunk is referred to as the gastrocolic trunk (GCT). If a common trunk is not formed by the ARCV and RGEV, there is no GCT. Even where the anterior superior pancreaticoduodenal vein (ASPDV) and ARCV 
Table 1. Arterial System of the Right Colon.

\begin{tabular}{lc}
\hline \multicolumn{1}{c}{ Blood vessel/feature } & Frequency of presence \\
\hline ICA & $100 \%(165 / 165)$ \\
RCA & $28.5 \%(47 / 165)$ \\
MCA & $100 \%(165 / 165)$ \\
- Independent branching of right and left & $-4.2 \%(7 / 165)$ \\
branches of MCA & \\
\hline
\end{tabular}

Data is presented as \% (number)

ICA, ileocolic artery; RCA, right colic artery; MCA, middle colic artery.

Table 2. Venous System of the Right Colon.

\begin{tabular}{|c|c|c|c|}
\hline $\begin{array}{c}\mathrm{GCT}(+)(\mathrm{n}=139) \\
(84.2 \% \text { of cases })\end{array}$ & & $\begin{array}{l}\mathrm{GCT}(-)(\mathrm{n}=26) \\
(15.8 \% \text { of cases })\end{array}$ & \\
\hline ARCV 1 & 111 & RCV 1 & 14 \\
\hline ARCV 2 & 26 & RCV 2 & 1 \\
\hline ARCV 3 & 2 & \multirow{2}{*}{$\begin{array}{l}\text { MCV is the drainage } \\
\text { vein of the right colon }\end{array}$} & \multirow[t]{2}{*}{11} \\
\hline - MCV also flowing into GCT & 10 & & \\
\hline - ICV also flowing into GCT & 1 & & \\
\hline
\end{tabular}

ARCV, accessory right colic vein; MCV, middle colic vein; ICV, ileocolic vein; GCT, Henle's gastrocolic trunk.

have merged, if there is no common trunk with the RGEV, GCT is taken as not existent.

4. $M C V$

The vein which flows from around the hepatic flexure and forms a common trunk with a drainage vein of the middle transverse colon is referred to as the right branch of middle colic vein (MCV).

\section{Results}

\section{Arterial system (Table 1)}

Frequency of presence of ICA was $100 \%$, whereas that of RCA was $28.5 \%$. Frequency of the presence of MCA was $100 \%$, and of these, seven cases $(4.2 \%)$ had independent branching of the right and left branches.

\section{Venous system (Table 2)}

There were 139 cases (84.2\%) in which GCT was present. Of these, 111 cases (79.9\%) (Figure 1) had 1 ARCV, 26 cases (18.7\%) (Figure 2) had 2 ARCVs, and 2 cases (1.4\%) (Figure 3) had 3 ARCVs. There were 10 cases where the MCV was flowing into the GCT and 1 case that had the ileocolic vein (ICV) flowing in. Further, there were two cases with GCT formation that also had RCV.

There were 26 cases $(15.8 \%)$ with no GCT present. There were 14 cases with $1 \mathrm{RCV}$ and 1 case with $2 \mathrm{RCVs}$, with the rate of presence of RCV among all cases $9.1 \%$. Moreover, there were 11 cases which had no RCV and the right

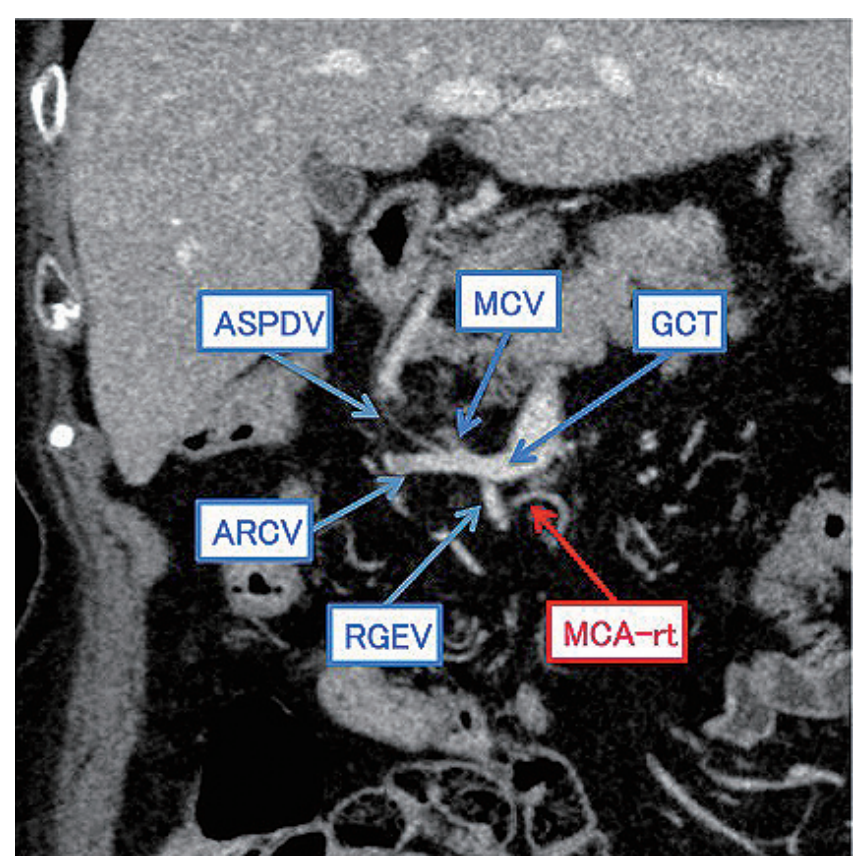

Figure 1. Examples of cases with one ARCV, where MCV also flows into GCT.

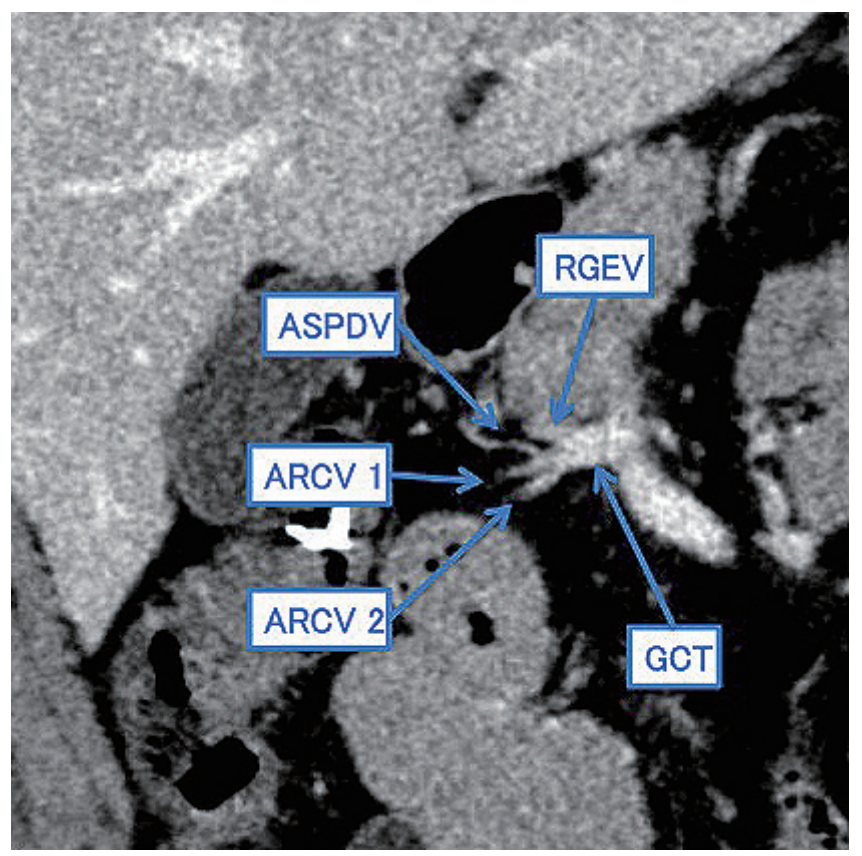

Figure 2. Examples of cases with two ARCVs.

branch of the MCV was the drainage vein for the right colon. The ASPDV flowed into the ARCV in 68 cases (41.2\%), into the RCV in 7 cases (4.2\%), into the right gastroepiploic vein (RGEV) in 38 cases $(23.1 \%)$, into the GCT in 44 cases $(26.7 \%)$, into the SMV in 5 cases $(3.0 \%)$, and unknown in 3 cases $(1.8 \%$ ) (Table 3 ). 


\section{Arteriovenous correlations}

Among the 139 cases with GCT present, the artery that accompanied the ARCV most frequently was the right branch of the MCA, that is, at 71.2\% (99/139) (Figure 4, 5), followed by the RCA at $19.4 \%(27 / 139)$ and with no accompanying arteries at $9.4 \%(13 / 139)$. Of the cases with no arteries accompanying the ARCV, the RCA was present in $15.4 \%(2 / 13)$. Of the 26 cases with no GCT, RCV existed in 15 cases. Of these, the artery accompanying the RCV was the right branch of the MCA in $66.6 \%$ of the cases $(10 / 15)$ (Figure 6,7 ) and the RCA in $33.3 \%$ of the cases $(5 / 15)$. The artery accompanying the ICV was the ICA in all cases (100\%). The accompanying vein in the 47 cases with RCA present was the ARCV in 27 cases, the RCV in 8 cases; the $\mathrm{MCV}$ in 1 case, and with no accompanying veins in 11 cases.

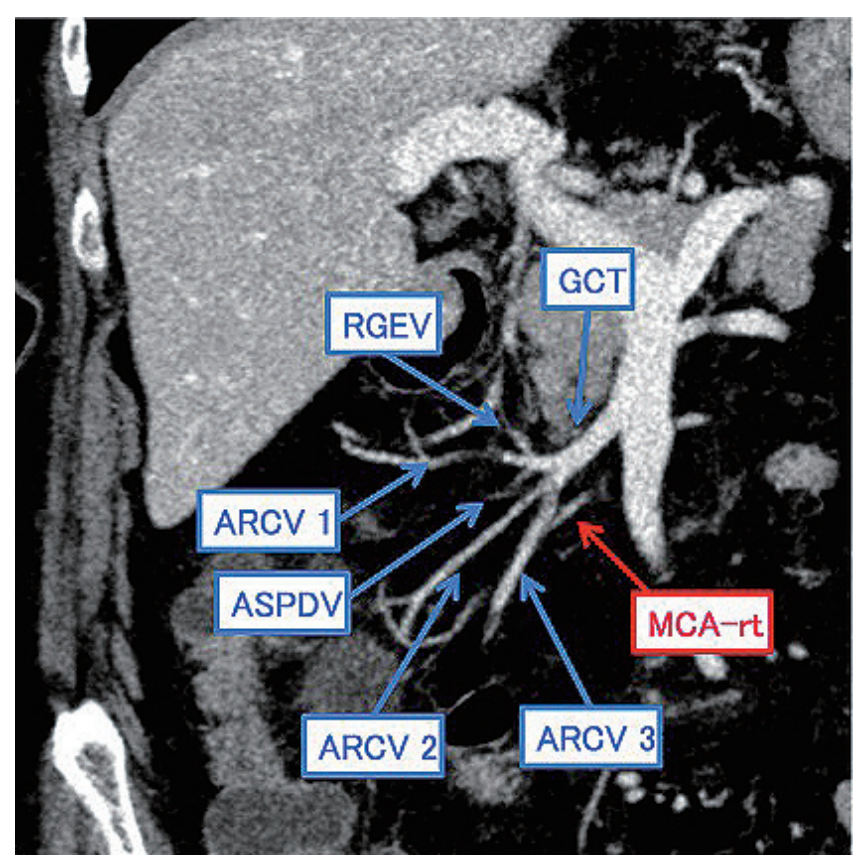

Figure 3. Examples of cases with three ARCVs.

\section{Discussion}

Previous studies have discussed the SMA or SMV, but with the progression in surgical techniques and image diagnoses, more detailed descriptions are now possible and thus have been detailed in this paper. However, practical anatomical knowledge is required for surgical procedures, and the details of the arteriovenous system and its correlations outlined here should be considered before gastroenterological surgery.

Surgery in respect of right colon cancer is one of the basic procedures used in gastroenterological surgery. With ileocecal resection, in which only the ileocolic arteries and veins are dissected, the branch formation of the blood vessels presents little variation; hence, complications during the surgical procedure are rare. However, with right hemicolectomy, there is a need to understand the complicated vascular branches in order to dissect the lymph nodes and to further identify and dissect the correct blood vessels. The branch morphologies of the RCV, MCV, and GCT are highly varied, and with both the head of the pancreas and the duodenum in close proximity, D3 lymphadenectomy is considered to be a highly difficult surgical technique.

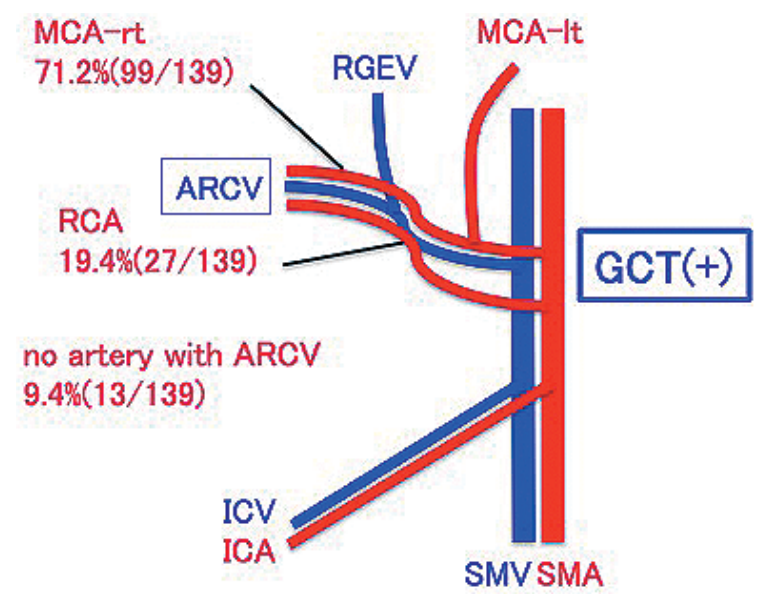

Figure 4. Correlation between $\mathrm{GCT}(+)$ and $\mathrm{ARCV}$ with arteries.

Table 3. Variations of Anterior Superior Pancreaticoduodenal Vein (ASPDV).

\begin{tabular}{lrrr}
\hline GCT $(+)(\mathrm{n}=139)(84.2 \%$ of cases $)$ & \multicolumn{2}{l}{ GCT $(-)(\mathrm{n}=26)(15.8 \%$ of cases $)$} \\
\hline - Flowing into ARCV & $68(41.2 \%)$ & - Flowing into RCV & $7(4.2 \%)$ \\
- Flowing into RGEV & $19(11.6 \%)$ & - Flowing into RGEV & $19(11.5 \%)$ \\
- Flowing into GCT & $44(26.7 \%)$ & & \\
- Flowing into SMV & $5(3.0 \%)$ & & \\
- Unknown & $3(1.8 \%)$ & & \\
\hline
\end{tabular}

Data is presented as number (\%)

ARCV, accessory right colic vein; RCV, right colic vein; RGEV, right gastroepiploic vein; GCT, Henle's gastrocolic trunk; SMV, superior mesenteric vein. 


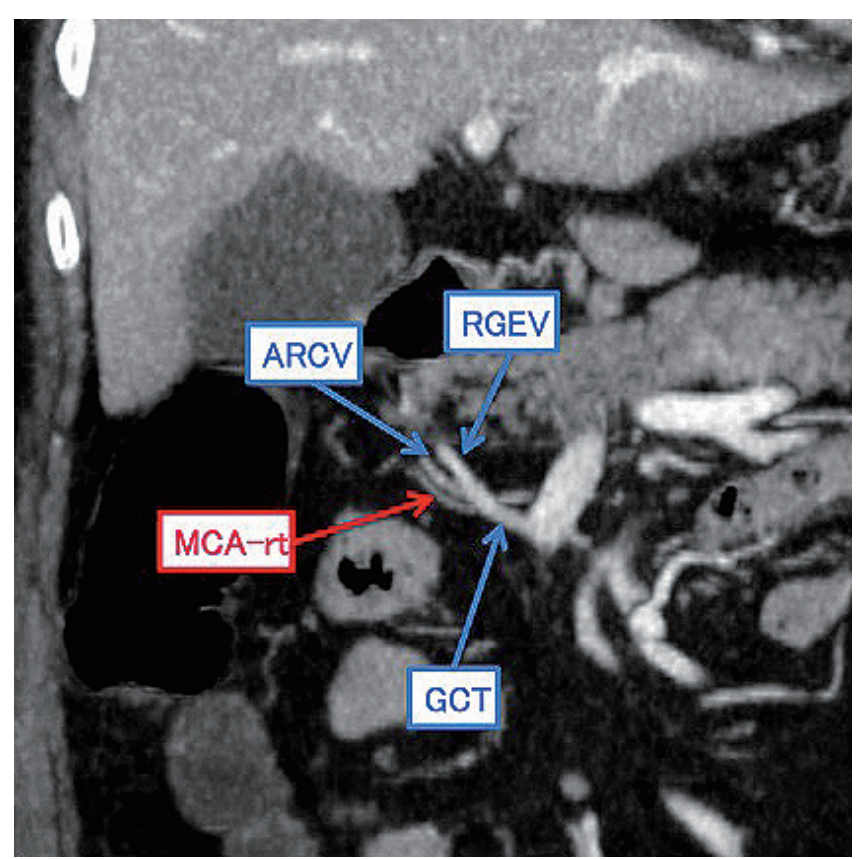

Figure 5. Examples of cases where the right branch of MCA accompanies GCT(+) and ARCV.

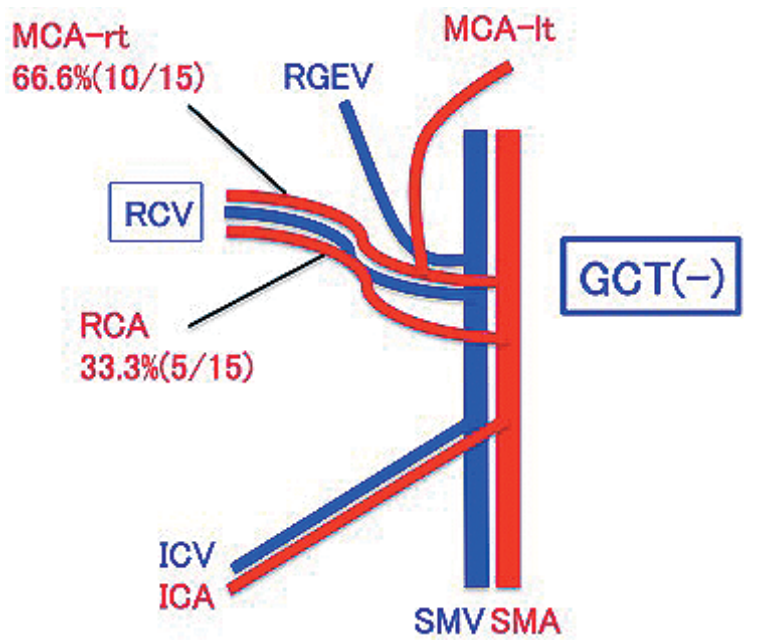

Figure 6. Correlation between $\mathrm{GCT}(-)$ and RCV with arteries.

According to the data from the Japanese National Clinical Database in 2015[28], the surgical mortality rate for right colon resection was slightly higher than that of low anterior resection for rectal cancer (at 1.3\% and $0.3 \%$, respectively). Although the exact reasons are unclear, we must take the complicated vascular branching into consideration and pay attention not to injury blood vessels during the surgery.

Multiple studies show that frequency of the presence of RCA is approximately $30 \%[22,24-26]$, which is in line with the results of this study. Although, one study reports that the MCA forms the common trunk in approximately $80 \%$ of cases[21], this particular morphology was more common in

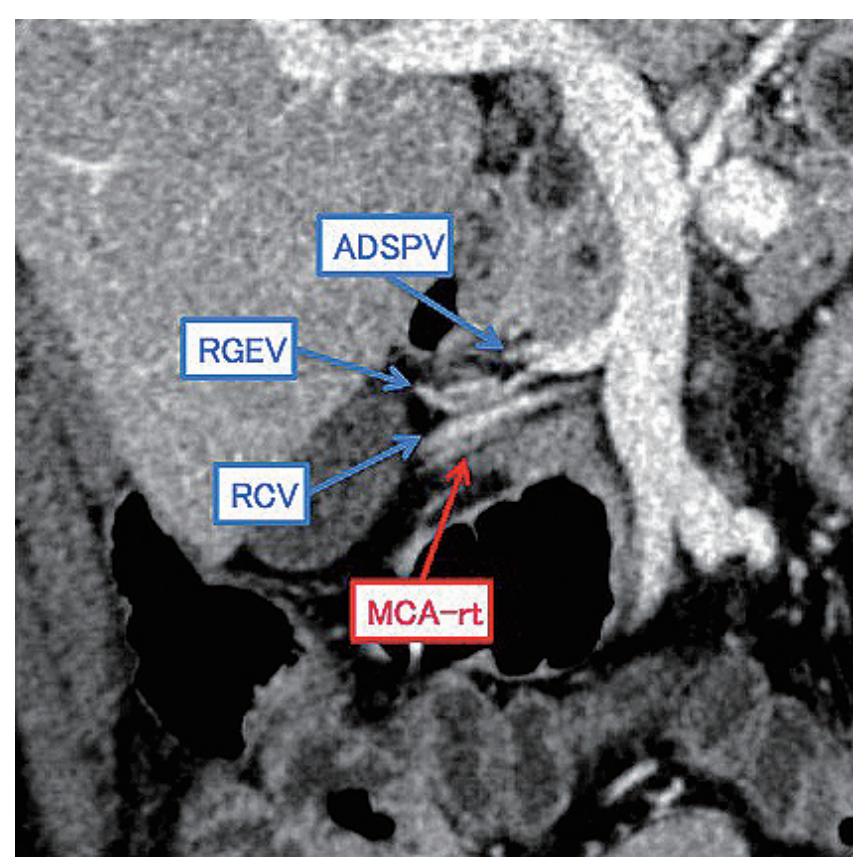

Figure 7. Examples of cases where the right branch of MCA accompanies GCT(-) and RCV.

this study, at over $90 \%$. Additionally, the frequency of RCV is reported to be approximately $20 \%-30 \%[8,22]$, but our results showed an incidence of less than $10 \%$. This may be due to the definition of RCV differing between reports. As described above, we defined RCV as an independent branch from the SMV, although there are some reports which include a branch at the base of the ICV in RCV[7]. Other studies report the frequency of presence of GCT as between $70 \%$ and $80 \%$ and frequency of MCV flowing into the GCT at approximately $10 \%[8,10,14,15,26]$.

Irrespective of GCT occurring, approximately $70 \%$ of the arteries accompanying the drainage vein from the right colon (ARCV or RCV) were found to be the right branch of the MCA; in approximately $20 \%-30 \%$ of the cases, it was the RCA; however, there were also cases when there were no accompanying veins or arteries. Moreover, in this study, in order to simplify the branching pattern, the ASPDV was not considered (as it flows into various branches, such as the ARCV, RCV, RGEV, GCT, or SMV); hence, including the ASPDV in this study, it would only further complicate branch formation descriptions.

As we have shown, there are many variations in the morphology of the right colon vascular anatomy, and there is a preoperative imperative to consider the individual vascular system of each patient. Pre-surgery contrast-enhanced CT imaging is a useful tool; however, there are several limitations. For example, there are small veins which are not detected by a contrast-enhanced CT; arteries and veins may not be clearly depicted depending on the timing of contrast agent injection; and a dilated intestinal tract may cause 
blood vessels (particularly veins) to retract during CT colonography, which also reduces clarity of depiction in the scans. Currently, in real practice, even if preoperative CT images are referred to, vascular recognition is determined ultimately based on the findings intraoperatively, and it is thought that the findings are sometimes different from preoperative expectations.

In this study, particular attention was given to the arteries that accompany the ARCV. As the ARCV is dissected at the pancreas head, it must be dissected carefully. The ARCV frequently merges with the anterior pancreaticoduodenal vein which is relatively short in length, although the accompanying artery may cover a longer distance, going beyond the SMV to the SMA. In this study, analysis showed that many cases occur in conjunction with the right branch of the MCA. According to the definition of D3 lymph node dissection, the MCA is completely dissected for cancer of the distal ascending colon; in the meantime, the ARCV is required to divide in most cases based on our research. There is room for debate regarding whether D3 lymph node dissection should be performed mostly on veins before the SMV, or only used on the arteries up to the SMA. However, the anatomical findings reported here may prove highly beneficial during right hemicolectomies for colon cancer.

This study has some limitations, including its singleinstitutional and retrospective nature. In addition, we did not confirm CT findings intraoperatively. We expect that further study will clarify the differences between the CT findings and the intraoperative findings.

\section{Conclusion}

The ARCV existed in most cases, and approximately $70 \%$ of feeding artery accompanying the ARCV or the RCV was the right branch of the MCA. In order to achieve safe and accurate surgery in respect to right colon cancer, we suggest that vascular branch formation should be considered preoperatively as standard practice.

\section{Acknowledgements}

The authors thank the work of past and present members of our department.

\section{Conflicts of Interest}

There are no conflicts of interest.

\section{Author Contributions}

NO, SY, and YO drafted the manuscript and provided the original pictures. All authors read and approved the final manuscript.

\section{Disclaimer}

Shigeki Yamaguchi is one of the Associate Editors of Journal of the Anus, Rectum and Colon and on the journal's
Editorial Board. He was not involved in the editorial evaluation or decision to accept this article for publication at all.

Approval by Institutional Review Board (IRB)

18-191, Saitama Medical University International Medical Center IRB

\section{References}

1. West NP, Morris EJ, Rotimi O, et al. Pathology grading of colon cancer surgical resection and its association with survival: a retrospective observational study. Lancet Oncol. 2008 Sep;9(9):857-65.

2. Hohenberger W, Weber K, Matzel K, et al. Standardized surgery for colonic cancer: complete mesocolic excision and central ligation--technical notes and outcome. Colorectal Dis. 2009 May; 11(4):354-64.

3. West NP, Hohenberger W, Weber K, et al. Complete mesocolic excision with central vascular ligation produces an oncologically superior specimen compared with standard surgery for carcinoma of the colon. J Clin Oncol. 2010 Jan;28(2):272-8.

4. Galizia G, Lieto E, De Vita F, et al. Is complete mesocolic excision with central vascular ligation safe and effective in the surgical treatment of right-sided colon cancers? A prospective study. Int J Colorectal Dis. 2014 Jan;29(1):89-97.

5. West NP, Kobayashi H, Takahashi K, et al. Understanding optimal colonic cancer surgery: comparison of Japanese D3 resection and European complete mesocolic excision with central vascular ligation. J Clin Oncol. 2012 May;30(15):1763-9.

6. Kobayashi H, West NP, Takahashi K, et al. Quality of surgery for Stage III colon cancer: comparison between England, Germany, and Japan. Ann Surg Oncol. 2014 Jun;21(3):398-404.

7. Micheles NA, Siddharth P, Kornblith PL, et al. The variant blood supply to the small and large intestine; its import in regional resections. J Int Coll Surg. 1963 Aug;39:127-70.

8. Yamaguchi S, Kuroyanagi H, Milsom JW, et al. Venous anatomy of the right colon: precise structure of the major veins and gastrocolic trunk in 58 cadavers. Dis Colon Rectum. 2002 Oct;45(10): 1337-40.

9. Ignjatovic D, Stimec B, Finjord T, Bergamaschi R. Venous anatomy of the right colon: three-dimensional topographic mapping of the gastrocolic trunk of Henle. Tech Coloproctol. 2004 Mar;8(1): 19-21; discussion 21-2.

10. Ignjatovic D, Spasojevic M, Stimec B. Can the gastrocolic trunk of Henle serve as an anatomical landmark in laparoscopic right colectomy? A postmortem anatomical study. Am J Surg. 2010;199 (2):249-54

11. Ignjatovic D, Sund S, Stimec B, Bergamaschi R. Vascular relationships in right colectomy for cancer: clinical implications. Tech Coloproctol. 2007 Sep;11(3):247-50.

12. Spasojevic M, Stimec BV, Fasel JF, et al. 3D relations between right colon arteries and the superior mesenteric vein: a preliminary study with multidetector computed tomography. Surg Endosc. 2011 Jun;25(6):1883-6.

13. Miyamoto R, Tadano S, Sano N, et al. The impact of threedimensional reconstruction on laparoscopic-assisted surgery for right-sided colon cancer. Wideochir Inne Tech Maloinwazyjne. 2017 Sep;12(3):251-6.

14. Ogino T, Takemasa I, Horitsugi G, et al. Preoperative evaluation of venous anatomy in laparoscopic complete mesocolic excision for 
right colon cancer. Ann Surg Oncol. 2014 Jun;21(3);429-35.

15. Jin G, Tuo H, Sugiyama M, et al. Anatomic study of the superior right colic vein: its relevance to pancreatic and colonic surgery. Am J Surg. 2006 Jan;191(1):100-3.

16. Sakaguchi T, Suzuki S, Morita Y, et al. Analysis of anatomic variants of mesenteric veins by 3-dimensional portography using multidetector-row computed tomography. Am J Surg. 2010 Jul;200 (1):15-22.

17. Lange JF, Koppert $\mathrm{S}$, van Eyck $\mathrm{CH}$, et al. The gastrocolic trunk of Henle in pancreatic surgery: an anatomo-clinical study. J Hepatobiliary Pancreat Surg. 2000 Aug;7(4):401-3.

18. Ibukuro K, Tsukiyama T, Mori K, Inoue Y. Peripancreatic veins on thin-section (3 mm) helical CT. AJR Am J Roentgenol. 1996 Oct; 167(4):1003-8.

19. Crabo LG, Conley DM, Graney DO, Freeny PC. Venous anatomy of the pancreatic head: normal CT appearance in cadavers and patients. AJR Am J Roentgenol. 1993 May;160(5):1039-45.

20. Zhang J, Rath AM, Boyer JC, et al. Radioanatomic study of the gastrocolic venous trunk. Surg Radiol Anat. 1994 Dec;16(4):413-8.

21. Negoi I, Beuran M, Hostiuc S, et al. Surgical anatomy of the superior mesenteric vessels related to colon and pancreatic surgery: a systematic review and meta-analysis. Sci Rep. 2018 Mar;8(1): 4184.

22. Hirai K, Yoshinari D, Ogawa $\mathrm{H}$, et al. Three-dimensional computed tomography for analyzing the vascular anatomy in laparoscopic surgery for right-sided colon cancer. Surg Laparosc Endosc
Percutan Tech. 2013 Dec;23(6):536-9.

23. Alsabilah J, Kim WR, Kim NK. Vascular structures of the right colon: incidence and variations with their clinical implications. Scand J Surg. 2017 Jun;106(2):107-15.

24. Murono K, Kawai K, Ishihara S, et al. Evaluation of the vascular anatomy of the right-sided colon using three-dimensional computed tomography angiography: a single-center study of 536 patients and a review of the literature. Int J Colorectal Dis. 2016 Sep;31(9):1633-8.

25. Shatari T, Fujita M, Nozawa K, et al. Vascular anatomy for right colon lymphadenectomy. Surg Radiol Anat. 2003 May;25(2):86-8.

26. Lee SJ, Park SC, Kim MJ, et al. Vascular anatomy in laparoscopic colectomy for right colon cancer. Dis Colon Rectum. 2016 Aug;59 (8):718-24.

27. Mori H, McGrath FP, Malone DE, Stevenson GW. The gastrocolic trunk and its tributaries: CT evaluation. Radiology. 1992 Mar;182 (3):871-7.

28. Kakeji Y, Udagawa H, Unno M, et al. Annual report of national clinical database in gastroenterological surgery 2015. Jpn J Gastroenterol Surg. 2017;50(2):166-76.

Journal of the Anus, Rectum and Colon is an Open Access journal distributed under the Creative Commons Attribution-NonCommercial-NoDerivatives 4.0 International License. To view the details of this license, please visit (https://creativ ecommons.org/licenses/by-nc-nd/4.0/). 